\title{
PRINCIPIA ETHICA E FILOSOFIA SOCIALE
}

\author{
GIORGIO LUNGHINI (*)
}

SunTO. - I Principia Ethica di G.E. Moore ebbero grande influenza sulla Società degli Apostoli e sul Circolo di Bloomsbury, e di qui sulla 'visione' di J.M. Keynes. Nelle parole di Schumpeter, «La Teoria Generale è il risultato finale della prolungata lotta di Keynes per rendere la sua visione della nostra era analiticamente operativa». Le Note conclusive sulla filosofia sociale verso la quale la Teoria generale potrebbe condurre sono infatti il più bell'esempio di una teoria economica intesa a fondare un disegno di politica economica, che indichi la retta via per fare il bene di tutti. I difetti più evidenti della società economica nella quale viviamo sono oggi gli stessi che Keynes denunciava nel 1936: l'incapacità ad assicurare una occupazione piena e la distribuzione arbitraria e iniqua della ricchezza e del reddito. Che cosa si dovrebbe fare, e si potrebbe fare, se davvero si condivide il giudizio che la disoccupazione e la disuguaglianza sono dei mali da guarire? Secondo Keynes si dovrebbero fare tre cose: una redistribuzione della ricchezza e del reddito, l'eutanasia del rentier, e una socializzazione di una certa ampiezza degli investimenti. È questo un disegno realistico e analiticamente ben fondato; tuttavia «La difficoltà sta nel fatto che i leaders capitalisti nella City e in parlamento non sono capaci di distinguere i nuovi strumenti e le misure per salvare il capitalismo da quello che loro chiamano bolscevismo».

$$
* * *
$$

Abstract. - The Principia Etbica of G.E. Moore highly influenced the Society of the Apostles and the Bloomsbury Circle, and hence the 'vision' of J.M. Keynes. In Schumpeter's words: "General Theory is the final outcome of Keynes's protracted struggle to make his vision of our era analytically operational". The Closing Notes on the social philosophy which the General Theory could lead to, represent in fact the best example of an economic theory intended to found a plan of economic policy, indicating the right way towards the greater good. Nowadays, the most apparent inadequacies of the economic society in which we live are the same as those reported by Keynes in 1936: the inability to provide full employment and the arbitrary and unfair distribution of wealth and income. What should be done, and could be done, if we all really share the view that unemployment and inequality are evil to be healed? According to Keynes,

(*) Istituto Lombardo Accademia di Scienze e Lettere; IUSS di Pavia. Italia. E-mail: giorgio.lunghini@iusspavia.it. 
three things should be done: a redistribution of wealth and income, the euthanasia of the Rentier, and a wide socialization of the investment. This is a realistic and analytically well-founded design; however, "The trouble is that capitalist leaders in the City and in parliament are not able to distinguish between the new instruments and measures to save capitalism and what they call Bolshevism".

Mi occuperò qui non delle teorie economiche dell'equità in generale, ma del più bell'esempio di una teoria economica intesa a fondare un disegno di politica economica, che indichi la retta via per fare il bene di tutti. La teoria è la Teoria generale di Keynes e il disegno è quello tracciato nel suo capitolo XXIV: Note conclusive sulla filosofia sociale verso la quale la teoria generale potrebbe condurre. Non ho invece il tempo per trattare come si deve la questione cui alludo: la dialettica tra being good e doing good e i rapporti tra Moore e Keynes, se non per ricordare la grande influenza che i Principia Ethica di Moore ebbero sulla Società degli Apostoli e sul Circolo di Bloomsbury, e di qui sulla 'visione' di Keynes. Nelle parole di Schumpeter «La Teoria Generale è il risultato finale della prolungata lotta di Keynes per rendere la sua visione della nostra era analiticamente operativa» ${ }^{1}$.

Il problema di fondo che Keynes affronta nel capitolo XXIV è lo stesso che dovremmo porci noi oggi: quale possa e debba essere il ruolo dello Stato nell'attuale situazione economica e sociale (per semplicità: dopo l'esaurimento del fordismo, la conseguente globalizzazione dell'economia, e la conseguente crisi ora in atto). È questo un tema che ricorre in tutta l'opera di Keynes, sin dalla Fine del laissez faire:

Dobbiamo tendere a separare quei servizi che sono tecnicamente sociali da quelli che sono tecnicamente individuali. L'azione più importante dello Stato si riferisce non a quelle attività che gli individui privati esplicano già, ma a quelle funzioni che cadono al di fuori del raggio d'azione degli individui, a quelle decisioni che nessuno prende se non le prende lo Stato. La cosa importante per il governo non è fare ciò che gli individui fanno già, e farlo un po' meglio o un po' peggio, ma fare

1 Si veda il saggio di Maria Michela Marzano, Keynes e Moore: il ruolo dei Principia Etbica in "My Early Belief", Storia del Pensiero Economico, 36 (1998). Per Keynes, vedi J.M. Keynes, The General Theory of Employment, Interest and Money, Londra, Macmillan, 1936, ristampa 2007; e La fine del laissez-faire e altri scritti economico-politici, a cura di G. Lunghini, Torino, Bollati Boringhieri, 1991. 
ciò che presentemente non si fa del tutto ... Il nostro problema è elaborare una organizzazione sociale che sia la più efficiente possibile, senza offendere le nostre nozioni di un soddisfacente sistema di vita.

I difetti più evidenti della società economica nella quale viviamo sono oggi gli stessi che Keynes denunciava nel 1936: l'incapacità a provvedere una occupazione piena e la distribuzione arbitraria e iniqua della ricchezza e del reddito. Questa persistenza patologica non trova spiegazioni convincenti nell'antropologia e nell'analisi economica reazionarie; mentre la possono spiegare la Teoria generale di Keynes e la miopia dei conservatori: «La difficoltà sta nel fatto che i leader capitalisti nella City e in parlamento non sono capaci di distinguere i nuovi strumenti e le misure per salvare il capitalismo da quello che loro chiamano bolscevismo». Per lunghi periodi il keynesismo può anche essere sembrato dominante, in forme più o meno oneste di spesa pubblica; e Keynes ha certamente autorizzato un intervento, diretto o indiretto, a sostegno della domanda effettiva e dunque dell'occupazione. L'idea era che soltanto by accident or design la domanda effettiva, per consumi e per investimenti, avrebbe coinciso con la produzione corrispondente al pieno impiego, e che perciò un intervento attivo del governo normalmente sarebbe stato necessario.

Tuttavia lo stesso Keynes non scommetteva sulla efficacia di politiche keynesiane: «Questa che io propongo è una teoria che spiega perché la produzione e l'occupazione siano così soggette a fluttuazioni: essa non offre una soluzione bella e pronta al problema di come evitare queste fluttuazioni e mantenere costantemente la produzione a livello ottimale». In un mondo dominato dalla finanza - che è il sottoprodotto delle attività di un casinò, dove i giochi nel migliore dei casi sono a somma zero - il moltiplicatore di una politica di spesa pubblica difficilmente sarà maggiore di uno, né potrà essere molto efficace un intervento di stimolo indiretto, mediante una riduzione del tasso d'interesse. In una situazione di deflazione, la conseguenza più probabile è la trappola della liquidità, non l'aumento degli investimenti privati.

Anziché il Keynes del breve periodo, è il Keynes radicale cui si dovrebbe pensare, anche perché ce ne sono le condizioni (non anche la volontà politica). Questo Keynes, il Keynes del capitolo XXIV della Teoria generale, non ha mai dominato in nessun governo e in nessuna università. Eppure vi si trovano analisi e disegni di estremo interesse. Che cosa si dovrebbe fare, e si potrebbe fare, se davvero si condivide il 
giudizio che i mali da guarire sono una distribuzione arbitraria e iniqua della ricchezza e del reddito e l'incapacità ad assicurare la piena occupazione? Il disegno di Keynes è articolato in tre punti.

La premessa del primo è la negazione della tesi ancora oggi corrente, secondo la quale l'accumulazione del capitale dipenderebbe dalla propensione al risparmio individuale, e che dunque in larga misura l'accumulazione di capitale dipende dal risparmio dei ricchi, la cui ricchezza risulta così socialmente legittimata. Proprio la Teoria generale mostra invece che, sino a quando non vi sia piena occupazione, l'accumulazione del capitale non dipende affatto da una bassa propensione a consumare, ma ne è invece ostacolata. Il risparmio disponibile presso le istituzioni finanziarie, d'altra parte, è maggiore di quello necessario, così che una redistribuzione del reddito intesa ad aumentare la propensione media al consumo potrebbe favorire l'accumulazione del capitale. Il luogo comune, secondo cui le imposte di successione provocherebbero una riduzione della ricchezza capitale del paese, è infondato. Oltre che garantire il principio (liberale) dell'eguaglianza dei punti di partenza, alte imposte di successione favorirebbero l'accumulazione di capitale, anziché frenarla. Dunque:

1. Nelle condizioni contemporanee l'aumento della ricchezza, lungi dal dipendere dall'astinenza dei ricchi, come in generale si suppone, è probabilmente ostacolato da questa. Viene quindi a cadere una delle principali giustificazioni sociali della grande disuguaglianza delle ricchezze ... Per mio conto, ritengo che vi siano giustificazioni sociali e psicologiche per rilevanti disuguaglianze dei redditi e delle ricchezze, ma non per disparità tanto grandi quanto quelle oggi esistenti. Vi sono pregevoli attività umane che richiedono il movente del guadagno e l'ambiente del possesso privato della ricchezza affinché possano esplicarsi completamente. Inoltre, l'esistenza di possibilità di guadagni monetari e di ricchezza privata può istradare entro canali relativamente innocui pericolose tendenze umane, le quali, se non potessero venir soddisfatte in tal modo, cercherebbero uno sbocco in crudeltà, nel perseguimento sfrenato del potere e dell'autorità personale e in altre forme di autopotenziamento. È meglio che un uomo eserciti la sua tirannia sul proprio conto in banca che sui suoi concittadini ... Ma per stimolare queste attività e per soddisfare queste tendenze non è necessario che le poste del gioco siano tanto alte quanto adesso. Poste assai inferiori serviranno ugualmente bene, non appena i giocatori vi si saranno abituati. Però non deve confondersi il compito di tramutare la natura umana col compito di trattare la natura umana medesima. Sebbene nella repubblica ideale sarebbe insegnato, ispirato o consigliato agli uomini di non interessarsi affatto alle 
poste del gioco, può essere pur tuttavia saggia e prudente condotta di governo consentire che la partita si giochi, sia pure sottoponendola a norme e limitazioni, fino a quando la media degli uomini, o anche soltanto una sezione rilevante della collettività, sia di fatto dedita tenacemente alla passione del guadagno monetario.

Il secondo passo del ragionamento di Keynes riguarda il saggio di interesse. La giustificazione normalmente addotta per un saggio di interesse moderatamente alto è la necessità di incentivare il risparmio, nell'infondata speranza di generare così nuovi investimenti e nuova occupazione. È invece vero, a parità di ogni altra circostanza, che gli investimenti sono favoriti da saggi di interesse bassi; così che sarà opportuno ridurre il saggio di interesse in maniera tale da rendere convenienti anche investimenti a redditività differita e bassa agli occhi del contabile, quali normalmente sono gli investimenti ad alta reddività sociale. Di qui la cicuta keynesiana, di straordinaria attualità: "l'eutanasia del rentier".

2. Lo stato di cose [sopra descritto] sarebbe del tutto compatibile con un certo grado di individualismo, tuttavia significherebbe l'eutanasia del rentier e di conseguenza l'eutanasia del potere oppressivo e cumulativo del capitalista di sfruttare il valore di scarsità del capitale. Oggi l'interesse non rappresenta il compenso di nessun sacrificio genuino, come non lo rappresenta la rendita della terra. [...] Potremmo dunque mirare in pratica (non essendovi nulla di tutto ciò che sia irraggiungibile) a un aumento del volume di capitale finché questo non fosse più scarso, cosicché l'investitore senza funzioni non riceva più un premio gratuito: e a un progetto di imposizione diretta tale da permettere che l'intelligenza e la determinazione e l'abilità del finanziere, dell'imprenditore et hoc genus omne (i quali certamente amano tanto il loro mestiere che il loro lavoro potrebbe ottenersi a molto minor prezzo che non attualmente) siano imbrigliate al servizio della collettività, con una ricompensa a condizioni ragionevoli.

Keynes aggiunge qui un corollario oggi blasfemo: «Rimarrebbe da decidere in separata sede su quale scala e con quali mezzi sia corretto e ragionevole chiamare la generazione vivente a restringere il suo consumo in modo da stabilire, nel corso del tempo, uno stato di benessere per le generazioni future». Infine, circa il ruolo dello Stato:

3. Lo Stato dovrà esercitare un'influenza direttiva circa la propensione a consumare, in parte mediante il suo schema di imposizione 
fiscale, in parte fissando il saggio di interesse e in parte, forse, in altri modi. Per di più, sembra improbabile che l'influenza della politica bancaria sul saggio di interesse sarà sufficiente da sé sola a determinare un ritmo ottimo di investimento. Ritengo perciò che una socializzazione di una certa ampiezza dell'investimento si dimostrerà l'unico mezzo per consentire di avvicinarci all'occupazione piena; sebbene ciò non escluda necessariamente ogni sorta di espedienti e di compromessi coi quali la pubblica autorità collabori con la privata iniziativa ... Non è la proprietà degli strumenti di produzione che è importante che lo Stato si assuma. Se lo Stato è in grado di determinare l'ammontare complessivo dei mezzi dedicati a aumentare gli strumenti di produzione e il saggio base di remunerazione per coloro che li possiedono esso avrà compiuto tutto quanto è necessario. Inoltre le necessarie misure di socializzazione possono essere introdotte gradualmente e senza apportare una soluzione di continuità nelle tradizioni generali della società.

Proporre queste tre ricette (redistribuzione della ricchezza e del reddito, eutanasia del rentier e una socializzazione di una certa ampiezza dell'investimento) come strumenti per combattere la disoccupazione e l'ineguaglianza può sembrare una predica. Esse si reggono invece su analisi difficili da liquidare, tanto che il problema viene spesso rimosso definendo la disoccupazione e l'ineguaglianza come fenomeni "naturali". Citando un aforisma di Paul Valery, Keynes ricorda che i conflitti politici distorcono e disturbano nella gente il senso di distinzione tra questioni di importanza e questioni di urgenza, e che dunque il cambiamento economico di una società è cosa da realizzare lentamente. Il cambiamento economico di una società è un processo lento, poiché richiede consenso politico circa un diverso modello di società, diverso circa la strada da prendere.

Tutto sommato, i tempi di Keynes dovevano essere molto più vivaci e progressisti dei nostri, se Keynes giudicava la teoria che ho riassunto sopra «moderatamente conservatrice nelle conseguenze che implica». Keynes sapeva bene che il suo manifesto era, se non rivoluzionario, oltraggiosamente radicale («Suggerire un'azione sociale per il bene pubblico alla City di Londra è come discutere L'origine delle specie con un vescovo sessant'anni fa»). Perciò spiegava che l'allargamento delle funzioni di governo da lui predicato, mentre sarebbe sembrato a un pubblicista del secolo XIX o a un finanziere americano contemporaneo una terribile usurpazione ai danni dell'individualismo, era da lui difeso «sia come l'unico mezzo attuabile per evitare la distruzione com- 
pleta delle forme economiche esistenti, sia come la condizione di un funzionamento soddisfacente dell'iniziativa individuale». Timore che sembra evocare lo spettro della rovina comune prefigurato dall'altro Manifesto.

L'assunzione di questa prospettiva era imposta, per il Keynes del '36, anche da importanti e lungimiranti considerazioni politiche: «Il mondo non tollererà ancora per molto tempo la disoccupazione, che è associata, inevitabilmente associata, con l'individualismo capitalista d'oggigiorno». L'assunzione di questa stessa prospettiva sarebbe inoltre più favorevole alla pace di quanto non sia un sistema teso alla conquista dei mercati altrui. Se le nazioni imparassero a costituirsi una situazione di piena occupazione mediante la loro politica interna, non vi sarebbero più ragioni economiche per contrapporre l'interesse di un paese a quello dei suoi vicini:

Il commercio internazionale cesserebbe di essere quello che è ora, ossia un espediente disperato per preservare l'occupazione interna forzando le vendite sui mercati esteri e limitando gli acquisti - metodo che, se avesse successo, sposterebbe semplicemente il problema della disoccupazione sul vicino che ha la peggio nella lotta - ma sarebbe uno scambio volontario e senza impedimenti di merci e servizi, in condizioni di vantaggio reciproco.

Nella chiusa della Teoria generale Keynes si chiede se l'avverarsi di queste idee sia speranza visionaria o se gli interessi che esse frustreranno non saranno più forti di quelli che esse promuoveranno. Inguaribile ottimista, Keynes si risponde che

Il potere degli interessi costituiti è assai esagerato in confronto con la progressiva estensione delle idee. Non però immediatamente, ... giacché nel campo della filosofia economica e politica non vi sono molti sui quali le nuove teorie fanno presa prima che abbiano venticinque o trent'anni di età, cosicché le idee che funzionari di Stato e uomini politici e perfino gli agitatori applicano agli avvenimenti correnti non è probabile che siano le più recenti. Ma presto o tardi sono le idee, non gli interessi costituiti, che sono pericolose sia in bene che in male.

Fatto il conto delle generazioni tra il ' 36 e oggi, è dunque tempo che gli uomini della pratica, i quali si credono liberi da qualsiasi influenza intellettuale, scoprano come vivo questo economista defunto. Vi tro- 
veranno almeno una risposta analoga a quella che il gatto, che aveva lunghi unghioli e tanti denti, dà ad Alice nel paese delle meraviglie. Alice aveva chiesto al gatto: "Potrebbe dirmi, per favore, che strada dovrei prendere?". La risposta del gatto, che aveva lunghi unghioli e tanti denti, fu: "Dipende molto da dove vuoi andare". 